\title{
Desempenho da marcha de idosos praticantes de psicomotricidade
}

\author{
Walking performance of elderly practitioners of psychomotricity \\ Desempeño de la marcha de ancianos practicantes de la psicomotricidad
}

\section{Sarah Lins dos Santos', Maria Júlia Guimarães Oliveira Soares', Eduardo Ravagni", Marta Miriam Lopes Costa', Maria das Graças Melo Fernandes'}

' Universidade Federal da Paraíba, Programa de Pós-Graduação em Enfermagem. João Pessoa-PB, Brasil. " Universidade de Brasília, Faculdade de Educação, Departamento de Teoria e Fundamentos. Brasília-DF, Brasil.

Submissão: 12-10-2012 Aprovação: 28-05-2014

\section{RESUMO}

Estudo quase experimental realizado com quinze idosos, desenvolvido na Clínica Escola de Fisioterapia da Universidade Federal da Paraíba, em 2011. Objetivou-se avaliar o desempenho da marcha de idosos praticantes de atividades psicomotoras. Foi realizada uma entrevista estruturada centrada em dados sociais, clínicos e funcionais, com o intuito de poder correlacionar diversas informações que sustentassem o objetivo do estudo. A média no desempenho cognitivo foi de 19,4, na primeira avaliação e de 23,2, na segunda. A média no desempenho de atividades relacionadas à marcha foi de 11,6, na primeira avaliação, e de 15,5, na segunda, logo após a prática de atividades psicomotoras. Constatou-se uma melhora significativa no desempenho das atividades relacionadas à marcha, com irregularidades que persistiram na altura e na continuidade do passo, bem como no desvio da linha média.

Descritores: Marcha; Avaliação; Idoso; Fisioterapia.

Quasi-experimental study conducted with fifteen elderlies, developed at the Physiotherapy Clinical School, Federal University of Paraíba, in 2011. The study aimed to evaluate the performance of gait in elderly practitioners of psychomotor activities. It was conducted a structured interview focusing on social, clinical and functional data, in order to be able to correlate various information which supported the aim of the study. The average cognitive performance was 19.4 at the first assessment and 23.2 in the second. The average performance of activities related to the gait was 11.6 at the first assessment, and 15.5 in the second, after the practice of psychomotor activities. It was found a significant improvement in the performance of activities related to the gait, with irregularities persisting in height and continuity of the step, as well as in the midline deviation.

Key words: March; Evaluation; Aged; Physical Therapy Specialty.

\section{RESUMEN}

Estudio casi experimental realizado con quince ancianos, desarrollado en la Clínica Escuela de Fisioterapia de la Universidad Federal de Paraíba, en 2011. Objetivó-se evaluar el desempeño de la marcha de ancianos practicantes de actividades psicomotoras. Con el fin de correlacionar informaciones diversas para apoyo al objetivo del estudio, se llevó a cabo una encuesta estructurada centrada en datos sociales, clínicos y funcionales. El rendimiento cognitivo promedio fue de 19,4 en la primera evaluación y 23,2 en el segundo. El rendimiento promedio de las actividades relacionadas con la marcha fue de 11,6 en la primera evaluación, y 15,5 en la segunda, después de la práctica de actividades psicomotoras. Se percibió una mejora significativa en el desempeño de actividades relacionadas con la marcha, persistiendo irregularidades en la altura y en la continuidad del paso, y en el desplazamiento de la línea media.

Palabras clave: Marcha; Evaluación; Anciano; Fisioterapia. 


\section{INTRODUÇÃO}

O corpo é uma unidade que se evidencia, especialmente no movimento. Agindo, percebendo ou sentindo, nós identificamos e nos relacionamos com os outros seres no mundo. Essa ação é realizada de acordo com um tempo e em relação a um espaço que, limitados ou facilitados por nossas propriedades psicomotoras, permitem a vivência da nossa unidade pessoal ${ }^{(1)}$.

As atividades do dia a dia provocam modificações no sistema de respostas emocionais e motoras que tornam possíveis diferentes níveis de interação com o mundo, adaptando o ser humano aos desafios da vida. Especificamente, a aprendizagem de habilidades motoras capacita-o a executar movimentos ligados a um determinado fim, como, por exemplo, os relacionados com a marcha.

No processo de envelhecimento, o ser humano evidencia o comprometimento de algumas estruturas fisiológicas importantes para o desempenho da marcha, especialmente a habilidade do sistema nervosos central de processar sinais vestibulares, visuais e proprioceptivos responsáveis pela manutenção do equilíbrio corporal, gerando, ocasionalmente, a instabilidade postural na posição bípede, o que altera o deslocamento do corpo em relação ao espaço ${ }^{(2-3)}$. Vale salientar que as modificações da marcha no idoso se processam tanto em relação aos fatores tanto fisiológicos quanto aos emocionais. As alterações na marcha podem ser percebidas ou constatadas pelas modificações motoras que retroalimentam as condições emocionais que o idoso elabora em relação às suas possibilidades psicomotoras, o que interfere na realização de algumas tarefas específicas.

Cerca de $30 \%$ a $40 \%$ dos idosos, com idade acima de 65 anos, podem apresentar alterações na marcha que facilitam as quedas, que são responsáveis por taxas significativas de atendimentos emergenciais e de hospitalização do idoso, $6 \%$ e $10 \%$ respectivamente ${ }^{(4)}$, além de, ocasionarem alterações funcionais, redução da autoestima e do domínio do esquema corporal, sendo, por isso, um fator de grande relevância epidemiologia, social e econômica em todo o mundo(5-6). A imagem do corpo ou a consciência que o ser humano tem de sua presença corporizada supera as fronteiras da anatomia. Assim, a marcha não pode ser compreendida sem se fazer referência às habilidades psicomotoras que compõem a imagem corporal. Essa imagem cristaliza as ações do sujeito por meio do esquema corporal, isto é, da propriedade que o ser humano tem do seu corpo como meio de interação e de comunicação consigo mesmo e com o mundo.

As possibilidades anatômicas e funcionais de cada corpo humano facilitam ou delimitam os deslocamentos do corpo no espaço e permitem que os movimentos corporais possam ser avaliados em relação às diversas variáveis. No presente estudo, ressalta-se a importância da velocidade, da amplitude e da segurança da cadência na marcha. Trata-se de variáveis importantes sobre a determinação do número de passos completados num determinado espaço e tempo, estabelecido pelo observador. Nessa atividade, avaliam-se os movimentos globais e segmentados, do equilíbrio estático e dinâmico, bem como do domínio dos deslocamentos ativos do corpo do idoso no espaço.
Portanto, o tempo que uma pessoa leva para caminhar uma determinada distância constitui a avaliação mais elementar em relação à marcha. Por outro lado, possibilita que se considerem outras variáveis dependentes desse deslocamento no espaço ${ }^{(7)}$. As modificações biomecânicas e fisiológicas não implicam unicamente um desempenho menos eficiente das habilidades motoras. Todavia, geram uma mudança qualitativa nos componentes subjacentes aos sistemas psicomotores envolvidos que controlam e ordenam os diferentes períodos da marcha.

Além disso, as atividades psicomotoras utilizadas no meio aquático facilitam a execução de movimentos que ordenam as sequências ou períodos que coordenam os deslocamentos corporais, relacionados à marcha. Nesse sentido, podem-se utilizar os princípios da psicomotricidade no meio aquático para ampliar a perspectiva de melhorias funcionais, especialmente, no que concerne a algumas variáveis inter-relacionadas. Nesse aspecto, destacam-se fatores como: equilíbrio, coordenação motora, deslocamentos posturais na água, controle dos movimentos globais e segmentados, domínio postural e esquema corporal. Isso acontece, porque a água atua como um meio facilitador da proposta motora que, direta ou indiretamente, estimula o aparecimento de reações psicoemocionais.

Diante dos argumentos expostos, uma análise detalhada da marcha é necessária quando se está diante dos possíveis fatores que podem ajudar a se compreender o desempenho motor de uma pessoa idosa. Através do fortalecimento da consciência e da imagem corporal como também dos elementos associados ao equilíbrio corporal e à coordenação motora, fortalece-se a corporeidade, tendo em vista que a segurança do meio aquático propicia a integração do corpo em movimento.

Nesse contexto, tentando desvelar os componentes fisiológicos e psicoemocionais que comprometem a qualidade da marcha em idosos comunitários, optou-se, neste estudo, por avaliar o desempenho da marcha de idosos praticantes de atividades psicomotoras.

\section{METODOLOGIA}

O estudo foi desenvolvido na Clínica Escola de Fisioterapia da Universidade Federal da Paraíba, localizada no município de João Pessoa - Paraíba. Trata-se de um estudo quase experimental, prospectivo, que envolveu quinze idosos comunitários. Para a escolha dos participantes, optou-se pela seleção dos indivíduos constantes numa lista de espera de clientes da Clínica Escola de Fisioterapia. Desses, apenas três atenderam aos critérios estabelecidos: ser idoso (idade de 60 anos ou mais); não participar de nenhuma atividade física ou fisioterapêutica; ser autossuficiente na execução das atividades da vida diária; ser capaz de ouvir, compreender e entender ordens simples; e ter consciência da orientação espacial e temporal.

Os critérios de exclusão do estudo levaram em consideração algumas condições exigidas pelo teste de equilíbrio Performance Oriented Mobility Assessment I (POMA) e pelo Miniexame de Estado Mental (MEEM), instrumentos de coleta de dados selecionados para o estudo pela confiabilidade e viabilidade, bem como por serem aceitos e validados no âmbito nacional e internacional ${ }^{(8-9)}$. $\mathrm{O}$ idoso selecionado não 
podia apresentar deficiência auditiva ou visual, distúrbios cognitivos, doença mental e dificuldade para movimentar as mãos como consequência de doenças reumáticas ou neurológicas. Também não podia apresentar um dos seguintes quadros: pressão arterial não controlada; dispneia aos mínimos esforços; uso de medicamentos psicotrópicos; e necessidade de apoio para realizar as atividades da vida diária.

Considerando os critérios de inclusão ora mencionados, como o número de participantes não atingia os propósitos do estudo, optou-se por uma segunda estratégia: a da seleção por rede. Com base nesse critério, os três primeiros idosos selecionados na lista de espera da Clínica Escola foram tomados como ponto de partida para a indicação de outros. Dessa forma, obteve-se uma amostra de conveniência e intencional composta por quinze idosos. A justificativa para se trabalhar com esse tipo de amostra seguiu o intuito do pesquisador de selecionar membros de uma comunidade mais acessível, que atendessem aos critérios estabelecidos para a pesquisa. Com base nesse critério, o pesquisador usa seu julgamento para selecionar os membros da população considerados boas fontes de informação ${ }^{(10)}$.

Todos os participantes assinaram o Termo de Consentimento Livre e Esclarecido, depois de especificar o objetivo do estudo. O projeto foi aprovado pelo Comitê de Ética em Pesquisa, do Centro de Ciências da Saúde, da Universidade Federal da Paraíba, sob o número 0163/11, conforme recomenda a Resolução 196/96 do Ministério da Saúde.

Os dados referentes a sexo, faixa etária, escolaridade, deficiência ou perturbação auditiva e/ou visual, prática de atividades físicas e história de quedas, foram coletados entre os meses de julho e setembro de 2011, mediante entrevista estruturada. Além disso, antes de iniciarem a primeira atividade psicomotora, todos os participantes foram submetidos ao MEEM e ao teste de POMA.

O MEEM constitui um instrumento composto de quatro categorias, as quais objetivam avaliar funções cognitivas específicas: orientação temporal, orientação espacial, registro de palavras, linguagem e praxia visuo construtiva. O escore do MEEM varia de 0 a 30 pontos, e valores mais baixos apontam para possível déficit cognitivo. Como esse teste sofre influência da escolaridade, foram utilizados os seguintes valores de referência ${ }^{(9)}$ : escore superior a 13 pontos, quando o idoso era analfabeto; e escore superior a 17 pontos, quando o idoso era alfabetizado.

O teste de POMA é utilizado para avaliar indivíduos da comunidade ou institucionalizados que tenham propensão a quedas ou que estejam sendo acompanhados para tratamento de déficit na mobilidade, sendo dividido em duas partes: uma avalia os fatores de risco de quedas em idosos, com base no número de incapacidades crônicas, e a outra avalia a marcha ${ }^{(8)}$. Esse instrumento é composto por 22 tarefas - 13 referentes ao equilíbrio e nove relacionadas à marcha. No presente estudo, foi utilizado apenas o teste de marcha. $\mathrm{Na}$ avaliação da marcha por meio desse teste, a pessoa pode ser assistida pelo examinador que, durante essa atividade, deve permanecer próximo a mesma para prevenir quedas devido a possíveis desequilíbrios. Os componentes avaliados são: iniciação da marcha; altura do passo; comprimento do passo; simetria de passo; continuidade do passo; desvio da linha média; estabilidade do tronco; base de apoio durante as fases da marcha; giro durante a marcha. De acordo com os princípios que orientam o teste, a pontuação mais elevada indica uma qualidade de desempenho melhor do indivíduo testado. A pontuação máxima de desempenho totaliza 57 pontos, sendo 39 para o teste de equilíbrio e 18 para o teste de marcha ${ }^{(8)}$.

Concluída a etapa de avaliação, os participantes realizaram atividades psicomotoras no ambiente aquático e no solo, duas vezes por semana, no turno da manhã, em sessões de 40 minutos, durante oito semanas, totalizando 17 dias. Esse programa foi considerado de baixo impacto e baixa intensidade, tendo em vista as particularidades da clientela atendida. Como procedimentos de rotina, foram verificadas a pressão arterial, a frequência cardíaca e respiratória, antes e depois das sessões das atividades psicomotoras. Esse cuidado teve o propósito de desvelar as condições gerais de cada idoso e, consequentemente, verificar o nível de segurança para realizar as atividades psicomotoras a serem efetivadas na água e no solo. Os equipamentos utilizados nas referidas atividades foram: piscina térmica, tensiômetro, estetoscópio, cronômetro, rádio, flutuadores, fisioball, cones, cesta e bastões.

Para a análise dos dados, foi utilizada a estatística descritiva, apresentada na forma de média, desvio- padrão e valores mínimos e máximos para as variáveis numéricas. Foram aplicados critérios estatísticos para comparar os resultados do teste de POMA e do MEEM, especificamente, a avaliação da marcha. As variáveis demográficas, assim como o desempenho das intervenções foram analisadas por meio do teste ANOVA. Para analisar as variáveis não paramétricas, utilizou-se o teste de Friedman, e para verificar as interações entre variáveis quantitativas, a análise de correlação paramétrica de Pearson. A normalidade dos dados foi testada por meio do teste de Kolmogorov-Smirnov, e o nível de significância foi estabelecido em 5\% para todas as análises estatísticas.

\section{RESULTADOS}

Dos 15 idosos pesquisados, 86,7\% eram do sexo feminino; a idade mínima foi de 60 , e a máxima, de 75 anos. A média de idade foi de 65,6 anos - 67,5, para o sexo masculino, e 65,3 , para o sexo feminino. Do total de entrevistados, $46,6 \%$ encontravam-se na faixa etária de 60 a 65 anos, e 33,3\% tinham entre 66 e 71 anos, e $20 \%$ apresentavam idade entre 70 e 75 anos. Quanto ao grau de escolaridade, 46,6\% dos entrevistados possuíam quatro a oito anos de estudo, 33,3\% eram analfabetos e 20,0\% possuíam o ensino superior completo. No tocante aos aspectos clínicos, destacaram-se as doenças reumáticas, o que correspondeu a $86,7 \%$ da população pesquisada, 20,0\% apresentaram doenças cardíacas, e 66,7\% usavam medicamentos.

Quanto ao desempenho cognitivo na primeira avaliação, a pesquisa mostrou que $66,7 \%$ dos idosos apresentavam déficit cognitivo leve ou moderado. A média e o desvio-padrão do MEEM para a primeira avaliação situaram-se em 18,9 $\pm 4,6$. A pontuação 20 no teste foi alcançada por $26,6 \%$ dos idosos que estudaram quatro a oito anos. Na segunda avaliação, a média do MEEM foi de 23,2 $\pm 3,5$. Observou-se que $46,7 \%$ dos 
idosos avaliados apresentavam funções cognitivas íntegras, enquanto 53,3\% tinham déficit cognitivo leve ou moderado.

Quanto às avaliações do MEEM, não houve diferença significativa, no sexo masculino $(t=-0,33 ; \mathrm{gl}=1 ; \mathrm{p}=0,79)$, entre os que sofreram quedas $(\mathrm{t}=-0,46$ : $\mathrm{gl}=4 ; \mathrm{p}=0,67)$ nem quanto aos níveis de escolaridade $\left(\mathrm{F}_{(5,20)}=2,32 ; \mathrm{P}=0,08\right)$. Porém, ocorreu diferença significativa entre os que não sofreram queda $(\mathrm{t}=-3,96: \mathrm{gl}=9 ; \mathrm{p}=0,003$ ) e entre os idosos do sexo feminino $(t=-3,03 ; g l=12 ; p=0,01)$. Ressalta-se que o declínio cognitivo pode representar uma variável que influencia diretamente no risco de quedas em idosos ${ }^{(11)}$. Apesar disso, as baixas correlações encontradas entre o POMA e o escore do MEEM devem ser analisadas com cautela, pois o risco de quedas é influenciado por diversos fatores, como alterações visuais, paresias, parestesias, diminuição da flexibilidade e mobilidade corporal ${ }^{(11-12)}$. $O$ fato da correlação entre declínio cognitivo e risco de quedas ter apresentado índice de determinação entre $15 \%$ e $38 \%$ indica que tal variável está associada ao aumento do risco de quedas nos idosos.

No tocante à idade, não foram encontradas correlações significativas em nenhuma das avaliações do MEEM ( $r=-0,03$ : $r=-0,12$ com $p>0,05)$. Em relação ao desempenho da marcha, as variáveis que não demonstraram normalidade foram: altura do passo, comprimento do passo, simetria do passo, continuidade do passo, desvio da linha média, estabilidade do tronco, sustentação durante a marcha e giro durante a marcha. Apenas 49,3\% dos idosos conseguiram atingir o padrão de normalidade segundo o teste de POMA. A intervenção favoreceu positivamente na reorganização do desempenho da marcha, porém não conseguiu levar ao resultado desejado em relação às demais variáveis.

A média obtida pelos idosos na primeira avaliação da marcha foi de 14,9 pontos. O valor mínimo encontrado nesse teste foi de 12 pontos, e o máximo foi de17 pontos. O desvio-padrão para a avaliação do desempenho da marcha foi de 1,5. Em relação à segunda avaliação do desempenho da marcha, os idosos obtiveram uma média de 12,9, sendo o valor mínimo de 10, e o máximo de 15 pontos. O desvio-padrão obtido nessa avaliação do desempenho da marcha foi de 1,44 pontos.

Observou-se diferença significativa entre as duas avaliações do desempenho da marcha $(t=3,68 ; \mathrm{gl}=14 ; \mathrm{p}=0,0025)$. Não houve correlação significativa entre nenhum resultado do desempenho da marcha com o MEEM (primeira avaliação $r=0,40 ; p=0,14$ e segunda avaliação $r=0,22 ; p=0,42$ ). Também, não houve correlação entre a idade e os resultados da marcha (primeira avaliação $r=-0,02 ; p=0,95$ e segunda avaliação $r=-0,21 ; p=0,46)$.

Em relação às avaliações do desempenho da marcha, não houve diferença significativa, no sexo masculino $(t=1,00$; $\mathrm{gl}=1 ; \mathrm{p}=0,50)$ e entre os que sofreram quedas $(t=2,56$; $\mathrm{gl}=4 ; \mathrm{p}=0,06)$. Quanto aos níveis de escolaridade, houve diferença apenas entre os idosos com escolaridade entre quatro e oito anos de estudo $(t=3,23 ; \mathrm{gl}=7 ; \mathrm{p}=0,01)$. Também ocorreu diferença significativa entre os que não sofreram queda ( $t=2,63 ; \mathrm{gl}=9 ; \mathrm{p}=0,03$ ) e entre os idosos do sexo feminino $(t=3,54 ; \mathrm{gl}=12 ; \mathrm{p}=0,004)$. Todos apresentaram desempenho inferior na segunda avaliação da marcha.

\section{DISCUSSÃO}

A marcha bípede envolve alguns estados do movimento comumente associados às subtarefas que devem ser realizadas com o intuito de poder coordenar um eficiente comportamento práxico. Tais tarefas incluem a geração contínua de movimentos repetitivos dos membros superiores e inferiores, a fim de que haja progressão na execução dos deslocamentos ativos do corpo no espaço como também um processo de ajuste constante do equilíbrio. Portanto, o ser humano, especialmente o idoso, deve procurar adaptar seu corpo às condições que o ambiente apresenta, por meio de uma progressiva e eficiente coordenação de movimentos, tanto no início como no término de qualquer atividade. Esses movimentos são organizados pelo sistema nervoso central, mediante a sincronização da atividade específica de várias cadeias musculares ${ }^{(7)}$.

Ressalte-se que, do ponto de vista psicomotor, deve-se considerar que essa propriedade motora do movimento não traduz fielmente a intencionalidade do movimento humano. Como se sabe, toda atividade humana está impregnada de afetividade, e outorga sentido prático a qualquer realização motora. Nesse sentido, o objetivo do trabalho psicomotor é de assegurar a vivência do corpo na integração de três importantes dimensões; a motora-instrumental, a emocional-afetiva e a práxico-cognitiva.

Com base nesses princípios, foram desenvolvidas atividades psicomotoras, com o objetivo de promover a interação entre movimento, sensação e cognição. Essas atividades visaram assegurar um melhor desempenho na marcha, por meio da apropriação da imagem e do esquema corporal peculiar a cada idoso. O trabalho realizado na água e no solo não se restringiu apenas à realização de tarefas psicomotoras, previamente planejadas. Além disso, empregaram-se dinâmicas de grupo, brincadeiras lúdicas e jogos competitivos, como uma forma de poder trabalhar o corpo em sua totalidade, e não apenas nas atividades relacionadas ao desempenho da marcha. Buscou-se ampliar a participação do idoso na proposta de trabalho psicomotor, usando o ambiente aquático, com o intuito de poder diminuir o impacto das atividades planejadas. No entanto, após a intervenção, os participantes permaneceram com alterações nas seguintes variáveis: altura do passo, comprimento do passo, continuidade de passo, desvio da linha média e estabilidade do tronco.

Deve-se, entretanto, considerar que a avaliação orientada da marcha compreende as variáveis de distância, pois ela mensura o grau de normalidade de variáveis relacionadas ao desempenho da marcha. Isso significa dizer que o tempo de execução para gerar um fortalecimento muscular na região dos quadríceps e dos isquiotibiais foi curto, em relação ao padrão de normalidade do teste. Analisando-se os aspectos biomecânicos relacionados à marcha, observa-se que o comprimento do passo é caracterizado como uma distância linear entre dois eventos sucessivos acompanhados pela mesma extremidade durante a marcha. Esse parâmetro é afetado pelo comprimento da perna, pela altura, idade e pelo sexo ${ }^{(13-15)}$.

O comprimento do passo pode ser reduzido pelo aumento da atividade excêntrica do músculo quadríceps femoral durante 
a fase final de apoio, bem como pelo aumento da atividade excêntrica dos músculos isquiostibiais durante a fase final de balanço. Há relato de significativa correlação entre a força dos membros inferiores e a velocidade da marcha, evidenciando que a perda de força relacionada à idade era um dos fatores que causavam o declínio desse parâmetro entre os idosos ${ }^{(15)}$.

Supõe-se que, quanto menor o comprimento do passo, maior é a cadência para aumentar a velocidade da marcha. Isso explica o autocuidado do idoso ao desenvolver essa ação biomecânica. É importante enfatizar que a velocidade da marcha e o comprimento do passo diferem de pessoa para pessoa, porquanto os dados antropométricos do indivíduo, especialmente seu peso e sua altura, influenciam tanto na velocidade quanto no comprimento do passo ${ }^{(14)}$.

Nessa perspectiva, é pertinente a avaliar a psicomotricidade em idosos sedentários como forma de lhes proporcionar uma melhoria na força muscular, no equilíbrio postural e na promoção da saúde. Busca-se, principalmente, assegurar uma qualidade de vida melhor para esses idosos por meio da potencialização de suas capacidades motoras e, obviamente, de seus interesses e aptidões emocionais diante do trabalho corporal proposto.

É preciso levar em consideração que os comportamentos cultivados durante o percurso de vida têm influência direta na qualidade de vida da pessoa idosa. Apesar disso, pode-se afirmar que as atividades psicomotoras melhoram a autonomia do ser humano, independentemente da idade, redimensionando sua corporeidade.

Como limitação desta pesquisa, considera-se a não avaliação do sistema sensório-motor dos idosos investigados, pois esse sistema tem importante função para o desempenho da habilidade motora, sensitiva e cognitiva.

\section{CONCLUSÃO}

Pode-se considerar que o objetivo do estudo foi alcançado plenamente, visto que foi possível analisar detalhadamente a marcha do grupo de idosos avaliados durante o desempenho de atividades psicomotoras. O método utilizado para isso foi uma ferramenta apropriada. Quanto aos principais achados, ressalta-se que a média no desempenho de atividades relacionadas à marcha foi de 11,6, na primeira avaliação, e de 15,5, na segunda. Logo após a prática de atividades psicomotoras, constatou-se uma melhora significativa no desempenho das atividades relacionadas à marcha, em que persistiram irregularidades na altura e na continuidade do passo, bem como no desvio da linha média.

Apesar do curto intervalo de tempo, a intervenção psicomotora trouxe uma resposta positiva sobre o desempenho da marcha dos idosos participantes da pesquisa, mediante o fortalecimento muscular realizado no ambiente aquático que, devido ao seu baixo impacto, facilitou o desempenho funcional dos idosos. Diante disso, sugere-se a adoção da intervenção psicomotora tanto para a prevenção de incapacidade quanto para a reabilitação funcional do idoso. A psicomotricidade é uma área de estudo e de atuação profissional que, ao utilizar os recursos referentes ao movimento e à afetividade, objetiva estabelecer o equilíbrio necessário entre corpo, indivíduo e ambiente, na apropriação da corporeidade. Porém, essa intervenção deve ser planejada, visando melhorar a conduta motora, senso-perceptiva, cognitiva e emocional do idoso. Considerando isso, salienta-se que, por meio do reforço à autoestima e ao domínio corporal, assegura-se a adequação da funcionalidade corpórea, traduzida por meio da melhoria na qualidade de vida, a partir da projeção das possibilidades psicomotoras da pessoa idosa.

\section{REFERÊNCIAS}

1. Santos RAR, Nascimento CP, Biscoli MRA, Labadessa VM. Sexualidade na terceira idade: pense um pouco no próprio preconceito. Rev Olhar Científico. 2010;1(2):1-11.

2. Lopes MNSS, Passerini CG, Travensolo FT. Eficácia de um protocolo fisioterapêutica para equilíbrio em idosos institucionalizados. Semina Cienc Biol Saude. 2010;31(2):143-52.

3. Figliolino JAM, Morais TB, Berbel AM, Dal Corso S. Análise da influência do exercício físico em idosos com relação ao equilíbrio, a marcha e atividade de vida diária. Rev Bras Geriatr Gerontol. 2009; 12(2):227-38.

4. Tinetti ME, Baker DI, King M, Gottschalk M, Murphy TE, Acampora D, et al. Effect of dissemination of evidence in reducing injuries from falls. $N$ Engl J Med 2008;359(3):259-62.

5. Aruka AH, Silva JAMG, Navega MT. Análise da concordância entre instrumentos de avaliação do equilíbrio corporal em idosos. Rev Bras Fisioter. 2011;15(6):460-6.
6. Chianca TCM, Andrade CR, Albuquerque J, Wenceslau LCC, Tadeu LFR, Macieira TGR, et al. Prevalência de quedas em idosos cadastrados em um Centro de Saúde de Belo Horizonte-MG. Rev Bras Enferm. 2013;66(2):234-40.

7. O' Sullivan SB, Schmitz TJ. Fisioterapia: avaliação e tratamento. 5. ed. São Paulo: Manole; 2010.

8. Gomes GC. Translation, transcultural adaptation, and analysis of the psychometric properties of the "performance-oriented mobility assessment" (POMA) for a sample of Brazilian institutionalized elderly. [dissertation]. Campinas: Universidade Estadual de Campinas- UNICAMP; 2003.

9. Bertolucci PH, Brucki SMD, Campacci SR. O mini-exame do estado mental em uma população geral: impacto da escolaridade. Arq Neuropsiquiatr. 1994;52(1):1-7.

10. Pinheiro RM, Castro GC, Silva HHC, Nunes JMG. Comportamento do consumidor. Rio de Janeiro: FGV; 2011.

11. Marinho Junior C, Cavenaghi S, Marino LHC. Escalas de mensuração e modalidades fisioterapêuticas na 
reabilitação de pacientes com equilíbrio deficitário. Arq Ciênc Saúde. 2011;18(1):44-9.

12. Perracini MR, Fló CM. Fisioterapia: teoria e prática: funcionalidade e envelhecimento. Rio de Janeiro: Guanabara Koogan; 2011.

13. Menz HB, Lord SR, Fitzpatrick RC. Aged-related differences in walking stability. Age Ageing. 2003;32(2):137-42.
14. Kovacs CR. Aged-related changes in gait and obstacle avoidance capabilities in older adults: a review. J Appl Gerontol. 2005;24(1):21-34.

15. Abreu SS, Caldas CP. Velocidade de marcha, equilíbrio e idade: um estudo correlacional entre idosas praticantes e idosas não praticantes de um programa de exercícios terapêuticos. Rev Bras Fisioter. 2008;12(4):324-30. 